\title{
On the geometry of the continuous-time generalized algebraic Riccati equation arising in LQ optimal control*
}

\author{
Augusto Ferrante ${ }^{\dagger}$ and Lorenzo Ntogramatzidis ${ }^{\star}$
}

\begin{abstract}
In this paper we analyze the properties of the set of solutions of the generalized continuous algebraic Riccati equation from a geometric perspective. In particular, we study the relationship existing between the solutions of the generalized Riccati equation and the output-nulling subspaces of the underlying system. This analysis reveals the presence of a subspace that plays an important role in the solution of the related optimal control problem.
\end{abstract}

\section{INTRODUCTION}

The classic solution of the continuous-time regular infinite-horizon linear quadratic (LQ) problem is traditionally expressed in terms of a symmetric solution $X$ of the Riccati equation

$$
X A+A^{\mathrm{T}} X-(S+X B) R^{-1}\left(S^{\mathrm{T}}+B^{\mathrm{T}} X\right)+Q=0,
$$

where $A, Q \in \mathbb{R}^{n \times n}, B, S \in \mathbb{R}^{n \times m}, R \in \mathbb{R}^{m \times m}$ are such that the so-called Popov matrix $\Pi$ satisfies

$$
\Pi \stackrel{\text { def }}{=}\left[\begin{array}{cc}
Q & S \\
S^{\mathrm{T}} & R
\end{array}\right]=\Pi^{\mathrm{T}} \geq 0
$$

and $R$ is invertible. When $R$ is allowed to be singular, the corresponding LQ problem is called singular, see e.g. [13], [20], [17], [16], [14]. In particular, in [13] and [20] it was proved that an optimal solution of the singular LQ problem exists for all initial conditions if the class of controls is extended to include distributions. In [15] the constrained generalized continuous algebraic Riccati equation was defined, in analogy with the discrete case, by replacing the inverse of the matrix $R$ appearing in (1) with its Moore-Penrose pseudoinverse. To our best knowledge, the recent papers [9], [10] were the first attempts to relate this equation to singular LQ optimal control problems. Indeed, in [9], [10] it was shown that the existence of symmetric solutions of the constrained generalized continuous-time Riccati equation is equivalent to the existence of impulse-free solutions of the associated singular LQ problem from any initial condition. This means, in particular, that an optimal control can always be expressed as a state-feedback. Now that the connection between the constrained generalized continuous-time algebraic Riccati equation and the singular LQ problem has been explained,

*Partially supported by the Italian Ministry for Education and Research (MIUR) under PRIN grant n. 20085FFJ2Z "New Algorithms and Applications of System Identification and Adaptive Control" and by the Australian Research Council under the grant FT120100604.

${ }^{\dagger}$ Augusto Ferrante is with Dipartimento di Ingegneria dell'Informazione, Università di Padova, via Gradenigo, 6/B - 35131 Padova, Italy. augustoddei.unipd. it

${ }^{\star}$ Lorenzo Ntogramatzidis is with the Department of Mathematics and Statistics, Curtin University, Perth (WA), Australia. L. Ntogramatzidis@curtin.edu.au the important issue arises of analyzing the set of solutions of such equation and the relations of each of such solutions with the corresponding LQ control problem. Considerable effort has been devoted in providing a geometric characterization of the set of solutions of the discrete counterpart of this Riccati equation, see [18], [6], [7], [8]. A similar characterization for the continuous time generalized Riccati equation has never been considered.

Another reason for analyzing the geometric structure of the solutions of the generalized continuous-time Riccati equation is the fact that this equation is a particular case of a more general type of Riccati equation that arises in the literature that flourished in the past twenty years on stochastic optimal control, see e.g. [1], [2], [3], [11] and the references cited therein.

In this paper a geometric analysis is carried out on the structure of the symmetric solutions of the constrained generalized continuous-time algebraic Riccati equation. In particular, we will prove that the dynamics of the closedloop system can be divided into a part that depends on the particular solution $X$ considered, and one part which is independent of it. We also show that the latter dynamics, which is not necessarily stable, is confined to a reachability output nulling subspace, so that it does not contribute to the cost function. The spectrum associated with the reachable part of this dynamics can therefore be assigned without affecting the optimality of the cost. We show that the LQ optimal control problem may admit a stabilizing solution even in cases in which the generalized continuous-time Riccati equation does not admit a stabilizing solution. This new feature has no parallel in the regular LQ problems.

This is the conference version of a longer journal paper submitted by the same authors to Automatica.

\section{THE GENERALIZED RICCATI EQUATION AND LINEAR QUADRATIC OPTIMAL CONTROL}

Consider the state differential equation

$$
\dot{x}(t)=A x(t)+B u(t), \quad x(0)=x_{0} \in \mathbb{R}^{n} .
$$

where $A \in \mathbb{R}^{n \times n}$ and $B \in \mathbb{R}^{n \times m}$. The classic LQ problem can be stated as the problem of finding the control $u(t), t \geq 0$, that minimizes the quadratic performance index

$$
J\left(x_{0}, u\right)=\int_{0}^{\infty}\left[\begin{array}{ll}
x^{\mathrm{T}}(t) & u^{\mathrm{T}}(t)
\end{array}\right]\left[\begin{array}{cc}
Q & S \\
S^{\mathrm{T}} & R
\end{array}\right]\left[\begin{array}{l}
x(t) \\
u(t)
\end{array}\right] d t
$$

subject to (3), where $Q \in \mathbb{R}^{n \times n}, S \in \mathbb{R}^{n \times m}$ and $R \in \mathbb{R}^{m \times m}$ satisfy (2). We denote $\Sigma \stackrel{\text { def }}{=}(A, B, \Pi)$, which is sometimes referred to as Popov triple. When $R$ is positive definite, the 
optimal control (when it exists) does not include distributions. If $R$ is only positive semidefinite, the optimal solution can contain Dirac deltas and its derivatives. Important links exist between the existence of the solutions of the socalled generalized continuous algebraic Riccati equation $\operatorname{GCARE}(\Sigma)$

$$
X A+A^{\mathrm{T}} X-(S+X B) R^{\dagger}\left(S^{\mathrm{T}}+B^{\mathrm{T}} X\right)+Q=0,
$$

subject to the constraint

$$
\operatorname{ker} R \subseteq \operatorname{ker}(S+X B),
$$

and the non-impulsive optimal solutions of the infinitehorizon LQ problem, [9], [10]. The equation (5) along with the condition (6) is usually referred to as the constrained generalized continuous algebraic Riccati equation, and denoted by $\operatorname{CGCARE}(\Sigma)$.

The crucial difference between the discrete and the continuous time is that, whereas in the discrete time the existence of symmetric positive semidefinite solutions of the constrained generalized discrete algebraic Riccati equation is equivalent to the solvability of the infinite-horizon LQ problem, in the continuous time case this correspondence holds for the socalled regular solutions, i.e., the optimal controls of the LQ problem that do not contain distributions.

\section{PRELIMinARY RESUlts ON CGCARE $(\Sigma)$}

The purpose of this section is to provide a geometric characterisation for the set of solutions of the generalized continuous algebraic Riccati equation. Most of the results in the sequel hinge on the geometric concepts of output-nulling subspace and friend. These concepts are briefly recalled in Appendix A for the sake of completeness. We herein recall a standard linear algebra result that is used in the derivations of this paper.

Lemma 3.1: Consider $\Pi=\left[\begin{array}{cc}Q & S \\ S^{\top} & R\end{array}\right]=\Pi^{\top} \geq 0$. Then,

(i) $\operatorname{ker} S \supseteq \operatorname{ker} R$;

(ii) $S R^{\dagger} R=S$;

(iii) $S\left(I_{m}-R^{\dagger} R\right)=0$;

(iv) $Q-S R^{\dagger} S^{\top} \geq 0$.

In view of (i) in Lemma 3.1, (6) is equivalent to $\operatorname{ker} R \subseteq$ $\operatorname{ker}(X B)$. The following notation is used throughout the paper. First, let $G \stackrel{\text { def }}{=} I_{m}-R^{\dagger} R$ be the orthogonal projector that projects onto ker $R$. Moreover, we consider a non-singular matrix $T=\left[T_{1} \mid T_{2}\right]$ where $\operatorname{im} T_{1}=\operatorname{im} R$ and $\operatorname{im} T_{2}=\operatorname{im} G$, and we define $B_{1} \stackrel{\text { def }}{=} B T_{1}$ and $B_{2} \stackrel{\text { def }}{=} B T_{2}$. Finally, to any $X=X^{\mathrm{T}} \in \mathbb{R}^{n \times n}$ we associate the following matrices

$$
\begin{aligned}
Q_{X} & \stackrel{\text { def }}{=} Q+A^{\mathrm{T}} X+X A, \\
S_{X} & \stackrel{\text { def }}{=} S+X B, \\
K_{X} & \stackrel{\text { def }}{=} R^{\dagger} S_{X}^{\mathrm{T}}, \\
A_{X} & \stackrel{\text { def }}{=} A-B K_{X}, \\
\Pi_{X} & \stackrel{\text { def }}{=}\left[\begin{array}{cc}
Q_{X} & S_{X} \\
S_{X}^{\mathrm{T}} & R
\end{array}\right] .
\end{aligned}
$$

When $X=X^{\mathrm{T}}$ is a solution of $\operatorname{CGDARE}(\Sigma)$, then $K_{X}$ is the corresponding gain matrix, $A_{X}$ the associated closed-loop matrix.

Remark 3.1: A symmetric and positive semidefinite solution of the generalized discrete-time algebraic Riccati equation also solves the constrained generalized discretetime algebraic Riccati equation, [6]. This fact does not hold in the continuous time, i.e., not all symmetric and positive semidefinite solutions of $\operatorname{GCARE}(\Sigma)$ are also solutions of $\operatorname{CGCARE}(\Sigma)$.

\section{Characterization OF THE SOLUTIONS OF CGCARE}

Since $\Pi$ is assumed symmetric and positive semidefinite, we can consider a factorization of the form

$$
\Pi=\left[\begin{array}{cc}
Q & S \\
S^{\mathrm{T}} & R
\end{array}\right]=\left[\begin{array}{c}
C^{\mathrm{T}} \\
D^{\mathrm{T}}
\end{array}\right]\left[\begin{array}{ll}
C & D
\end{array}\right],
$$

where $Q=C^{\mathrm{T}} C, S=C^{\mathrm{T}} D$ and $R=D^{\mathrm{T}} D$. Let us define

$$
G(s) \stackrel{\text { def }}{=} C\left(s I_{n}-A\right)^{-1} B+D .
$$

Let $G^{\sim}(s) \stackrel{\text { def }}{=} G^{\mathrm{T}}(-s)$. The spectrum is defined as

$$
\Phi(s) \stackrel{\text { def }}{=} G^{\sim}(s) G(s) .
$$

It is easy to see that

$\Phi(s)=\left[\begin{array}{ll}B^{\mathrm{T}}\left(-s I_{n}-A^{\mathrm{T}}\right)^{-1} & I_{m}\end{array}\right]\left[\begin{array}{cc}Q & S \\ S^{\mathrm{T}} & R\end{array}\right]\left[\begin{array}{c}\left(s I_{n}-A\right)^{-1} B \\ I_{m}\end{array}\right]$.

We recall the following classical result.

Lemma 4.1: For any $X=X^{\mathrm{T}} \in \mathbb{R}^{n \times n}$, there holds

$$
\Phi(s)=\left[\begin{array}{ll}
B^{\mathrm{T}}\left(-s I_{n}-A^{\mathrm{T}}\right)^{-1} & I_{m}
\end{array}\right] \Pi_{X}\left[\begin{array}{c}
\left(s I_{n}-A\right)^{-1} B \\
I_{m}
\end{array}\right] .
$$

Proof: Let us define

$$
L_{X} \stackrel{\text { def }}{=} \Pi_{X}-\Pi=\left[\begin{array}{cc}
A^{\mathrm{T}} X+X A & X B \\
B^{\mathrm{T}} X & 0
\end{array}\right] .
$$

It is enough to prove that

$$
\left[\begin{array}{cc}
B^{\mathrm{T}}\left(-s I_{n}-A^{\mathrm{T}}\right)^{-1} & I_{n}
\end{array}\right] L_{X}\left[\begin{array}{c}
\left(s I_{n}-A\right)^{-1} B \\
I_{n}
\end{array}\right]=0 .
$$

Indeed

$$
\begin{aligned}
& {\left[\begin{array}{ll}
B^{\mathrm{T}}\left(-s I_{n}-A^{\mathrm{T}}\right)^{-1} & I_{n}
\end{array}\right] L_{X}\left[\begin{array}{c}
\left(s I_{n}-A\right)^{-1} B \\
I_{n}
\end{array}\right]} \\
& =-B^{\mathrm{T}}\left(s I_{n}+A^{\mathrm{T}}\right)^{-1}\left[\left(s I_{n}+A^{\mathrm{T}}\right) X-X\left(s I_{n}-A\right)\right]\left(s I_{n}-A\right)^{-1} B \\
& \quad-B^{\mathrm{T}}\left(s I_{n}+A^{\mathrm{T}}\right)^{-1} X B+B^{\mathrm{T}} X\left(s I_{n}-A\right)^{-1} B=0 .
\end{aligned}
$$

The following important result is the continuous-time counterpart of Theorem 3.1 in [6].

Theorem 4.1: Let $X=X^{\mathrm{T}}$ be a solution of $\operatorname{CGCARE}(\Sigma)$. Then, 
1) $W(s) \stackrel{\text { def }}{=} R^{\frac{1}{2}} R^{\dagger} S_{X}^{\mathrm{T}}\left(s I_{n}-A\right)^{-1} B+R^{\frac{1}{2}}$ is a square spectral factor of $\Phi(s)$;

2) $\operatorname{normrank} \Phi(s)=\operatorname{rank} R$

Now we show that, given a solution $X=X^{\mathrm{T}}$ of $\operatorname{GCARE}(\Sigma)$ :

(a) $\operatorname{ker} X$ is an output-nulling subspace for the quadruple $(A, B, C, D)$, i.e.,

$$
\left[\begin{array}{l}
A \\
C
\end{array}\right] \operatorname{ker} X \subseteq\left(\operatorname{ker} X \oplus 0_{p}\right)+\operatorname{im}\left[\begin{array}{l}
B \\
D
\end{array}\right] ;
$$

(b) the gain $K_{X}$ is such that $-K_{X}$ is a friend of $\operatorname{ker} X$, i.e.,

$$
\left[\begin{array}{l}
A-B K_{X} \\
C-D K_{X}
\end{array}\right] \operatorname{ker} X \subseteq \operatorname{ker} X \oplus 0_{p}
$$

In the case where $X=X^{\mathrm{T}}$ is the solution of $\operatorname{GCARE}(\Sigma)$ corresponding to the optimal cost, these properties are intuitive. Now we prove that the following stronger result holds.

Theorem 4.2: Let $X=X^{\mathrm{T}}$ be a solution of $\operatorname{GCARE}(\Sigma)$. Then, $\operatorname{ker} X$ is an output-nulling subspace of the quadruple $(A, B, C, D)$ and $-K_{X}$ is a friend of $\operatorname{ker} X$.

Proof: Since $X$ is a solution of $\operatorname{GCARE}(\Sigma)$, the Lyapunov equation

$$
X A_{X}+A_{X}^{\mathrm{T}} X+Q_{0 X}=0
$$

holds, where $Q_{0 X} \stackrel{\text { def }}{=} Q-S R^{\dagger} S^{\mathrm{T}}+X B R^{\dagger} B^{\mathrm{T}} X=C_{X}^{\mathrm{T}} C_{X} \geq 0$. Let $\xi \in \operatorname{ker} X$. If we multiply (9) to the left by $\xi^{\mathrm{T}}$ and to the right by $\xi$, we obtain $C_{X} \xi=0$, which proves that $\operatorname{ker} X \subseteq \operatorname{ker} C_{X}$. We multiply the same equation to the right by $\xi$, and we obtain $X A_{X} \xi=0$, which shows that $\operatorname{ker} X$ is $A_{X}$-invariant. We have proved that $\operatorname{ker} X$ is an $A_{X}$-invariant subspace contained in the null-space of $C_{X}$, and this shows that $\operatorname{ker} X$ is an outputnulling subspace for $(A, B, C, D)$ and $-K_{X}=-R^{\dagger} S_{X}$ is an associated friend.

We will show that the reachable subspace $\mathscr{R}_{\text {ker } X}^{\star}$ on the output-nulling subspace $\operatorname{ker} X$, coincides with the reachable subspace of the pair $\left(A_{X}, B G_{X}\right)$, that we denote by $\mathscr{R}_{0, X}$. Before we establish this fact, we first need to give some additional results on the solutions of $\operatorname{CGCARE}(\Sigma)$.

Lemma 4.2: Let $X=X^{\mathrm{T}}$ solve $\operatorname{CGCARE}(\Sigma)$ and let $\mathscr{R}_{0, X}$ denote the reachable subspace associated with the pair $\left(A_{X}, B G\right)$, i.e.,

$$
\mathscr{R}_{0, X} \stackrel{\text { def }}{=} \operatorname{im}\left[B G A_{X} B G A_{X}^{2} B G \quad \ldots A_{X}^{n-1} B G\right] .
$$

Let $C_{X} \stackrel{\text { def }}{=} C-D R^{\dagger} S_{X}^{\mathrm{T}}$. There holds

$$
\mathscr{R}_{0, X} \subseteq \operatorname{ker} C_{X} .
$$

The following result shows that the subspace $\mathscr{R}_{0, X}$ is independent of the particular solution $X=X^{\mathrm{T}}$ of
$\operatorname{CGCARE}(\Sigma)$, and such is also the spectrum of the closedloop matrix restricted to $\mathscr{R}_{0, X}$.

Theorem 4.3: Let $X=X^{\mathrm{T}}$ be a solution of $\operatorname{CGCARE}(\Sigma)$, and let $\mathscr{R}_{0, X}$ be defined by (10). Then,

- $\mathscr{R}_{0, X}$ is independent of $X$;

- $\left.A_{X}\right|_{\mathscr{R}_{0, X}}$ is independent of $X$.

As a consequence of this result, if the spectrum of the closedloop matrix restricted to $\mathscr{R}_{0, X}$ contains unstable eigenvalues, no solutions of $\operatorname{CGCARE}(\Sigma)$ can stabilize the closed loop. Notice that this issue does not arise in the standard case because, when $R$ is positive definite, matrix $G$ is zero, and therefore $\mathscr{R}_{0, X}=\{0\}$ for every symmetric solution $X=X^{\mathrm{T}}$ of $\operatorname{CARE}(\Sigma)$.

Theorem 4.4: Let $X=X^{\mathrm{T}}$ be a solution of $\operatorname{CGCARE}(\Sigma)$. Let $\mathscr{R}_{\operatorname{ker} X}^{\star}$ be the largest reachability subspace on $\operatorname{ker} X$. Then, $\mathscr{R}_{\text {ker } X}^{\star}=\mathscr{R}_{0, X}$.

Proof: Since $\mathscr{R}_{0, X}$ is the reachable subspace of the pair $\left(A_{X}, B G\right)$, it is the smallest $A_{X}$-invariant subspace containing $\operatorname{im}(B G)=B \operatorname{ker} D$. The reachability output-nulling subspace $\mathscr{R}_{\text {ker } X}^{\star}$ on the output-nulling subspace $\operatorname{ker} X$ is the smallest $(A+B F)$-invariant subspace containing $\operatorname{ker} X \cap B \operatorname{ker} D$, where $F$ be an arbitrary friend of $\operatorname{ker} X$, see Appendix A. The subspace $\mathscr{R}_{\operatorname{ker} X}^{\star}$ does not depend on the choice of the friend $F$, [19, Theorem 7.18]. In view of Theorem 4.2, $F=-K_{X}$ is a particular friend of $\operatorname{ker} X$. For this choice of $F$, we have $A+B F=A-B K_{X}=A_{X}$. Moreover, $\operatorname{ker} X \cap$ $B \operatorname{ker} D=B \operatorname{ker} D$, because the inclusion $\operatorname{ker} R \subseteq \operatorname{ker}(X B)$ implies $\operatorname{ker} X \supseteq B \operatorname{ker} D$.

\section{Stabilization}

So far we have shown that the spectrum of the closed-loop matrix $A_{X}$ restricted to the subspace $\mathscr{R}_{0, X}$ is independent of the particular solution $X=X^{\mathrm{T}}$ of $\operatorname{CGCARE}(\Sigma)$ considered. This means that the corresponding eigenvalues are present in the closed-loop independently of the solution $X=X^{\mathrm{T}}$ of $\operatorname{CGCARE}(\Sigma)$ considered. Since we have shown that $\mathscr{R}_{0, X}=$ $\mathscr{R}_{\text {ker } X}^{\star}$, it is always possible to find a matrix $L$ that assigns all the eigenvalues of the map $\left(A_{X}+B G L\right)$ restricted to $\mathscr{R}_{\operatorname{ker} X}^{\star}$, by adding a further term $B G L x(t)$ to the feedback control law. This operation does not change the value of the cost with respect to the one obtained by $u(t)=-K_{X} x(t)$, because this additional term only affects the part of the trajectory on $\mathscr{R}_{\text {ker } X}^{\star}$ which is output-nulling. In doing so it may stabilize the closed-loop. Indeed, since $\mathscr{R}_{0, X}$ is output-nulling with respect to the quadruple $(A, B, C, D)$, it is also output-nulling for the quadruple $\left(A-B K_{X}, B, C-D K_{X}, D\right)$, and two matrices $\Xi$ and $\Omega$ exist such that

$$
\left[\begin{array}{l}
A_{X} \\
C_{X}
\end{array}\right] R_{0, X}=\left[\begin{array}{c}
R_{0, X} \\
0
\end{array}\right] \Xi+\left[\begin{array}{l}
B \\
D
\end{array}\right] \Omega,
$$

where $R_{0, X}$ is a basis matrix of $\mathscr{R}_{0, X}$, see Appendix A. In order to find a feedback matrix which stabilizes the system, we solve (12) in $\Xi$ and $\Omega$, so as to find $L$ such that $\left[\begin{array}{l}A_{X}+B L \\ C_{X}+D L\end{array}\right] R_{0, X}=\left[\begin{array}{c}R_{0, X} \\ 0\end{array}\right] \Xi$, where the eigenvalues of $\Xi$ are the 
eigenvalues of the map $A_{X}+B L$ restricted to $\mathscr{R}_{0, X}$. We first compute the set of solutions of (12) in $\Xi$ and $\Omega$, i.e.,

$$
\left[\begin{array}{l}
\Xi \\
\Omega
\end{array}\right]=\left[\begin{array}{c}
\hat{\Xi} \\
\hat{\Omega}
\end{array}\right]+\left[\begin{array}{l}
H_{1} \\
H_{2}
\end{array}\right] K,
$$

where

$$
\left[\begin{array}{c}
\hat{\Xi} \\
\hat{\Omega}
\end{array}\right] \stackrel{\text { def }}{=}\left[\begin{array}{cc}
R_{0} & B \\
0 & D
\end{array}\right]^{\dagger}\left[\begin{array}{l}
A_{X} \\
C_{X}
\end{array}\right] R_{0}
$$

and $\left[\begin{array}{l}H_{1} \\ H_{2}\end{array}\right]$ is a basis matrix of $\operatorname{ker}\left[\begin{array}{cc}R_{0} & B \\ 0 & D\end{array}\right]$. Since $\mathscr{R}_{0, X}$ is an output-nulling reachability subspace, the pair $\left(\hat{\Xi}, H_{1}\right)$ is completely reachable. This implies that a matrix $K$ in (13) can always be found so that the eigenvalues of $\Xi$ are freely assignable (provided they come in complex conjugate pairs). Hence, we use such $K$ in (13) and then we compute $L=-\Omega R_{0}^{\dagger}$. This choice guarantees that only the eigenvalues of $A_{X}$ restricted to $\mathscr{R}_{0, X}$ get affected by the use of $L$.

Example 5.1: Consider the quadruple $A=\left[\begin{array}{ccc}1 & 1 & 1 \\ -3 & 1 & 0 \\ 1 & 0 & 0\end{array}\right]$, $B=\left[\begin{array}{ll}0 & 2 \\ 0 & 0 \\ 1 & 0\end{array}\right], Q=\left[\begin{array}{ccc}1 & 0 & -1 \\ 0 & 0 & 0 \\ -1 & 0 & 1\end{array}\right], S=\left[\begin{array}{cc}1 & 0 \\ 0 & 0 \\ -1 & 0\end{array}\right]$ and $R=\left[\begin{array}{ll}1 & 0 \\ 0 & 0\end{array}\right]$. One can directly verify that the only two solutions of $\operatorname{CGCARE}(\Sigma)$ are $X_{0}=0$ and $X_{1}=\operatorname{diag}\{0,0,2\}$. None of these two solutions is stabilizing. Indeed, the eigenvalues of the closed-loop matrix relative to $X_{0}$ are $\{1,1 \pm i \sqrt{3}\}$, while those of the one relative to $X_{1}$ are $\{-1,1 \pm i \sqrt{3}\}$. Thus, $\operatorname{CGCARE}(\Sigma)$ does not have a stabilizing solution. However, the infinite-horizon LQ problem admits an optimal solution. Consider for example $X=X_{0}$. We find $\mathscr{R}_{0, X_{0}}=\left\langle A_{X_{0}}, B G\right\rangle=\operatorname{im}\left[\begin{array}{ll}1 & 0 \\ 0 & 1 \\ 0 & 0\end{array}\right]$. Since this subspace does not depend on the particular solution of $\operatorname{CGCARE}(\Sigma)$, we have $\mathscr{R}_{0, X_{1}}=\mathscr{R}_{0, X_{0}}$. Since this subspace has dimension 2 , we can stabilize at most 2 eigenvalues in the closed-loop matrix without altering the cost. On the other hand, since $A_{X_{0}}$ has three unstable eigenvalues, we cannot obtain a stabilizing feedback from $X_{0}$. We show that we can obtain a stabilizing feedback from $X=X_{1}$. Using (13) we compute $\hat{\Xi}=\left[\begin{array}{cc}1 / 5 & 1 / 5 \\ -3 & 1\end{array}\right]$ and $\hat{\Omega}=\left[\begin{array}{cc}0 & 0 \\ 2 / 5 & 2 / 5\end{array}\right]$. A basis for the null-space of $\left[\begin{array}{rr}R_{0, X} & B \\ 0 & D\end{array}\right]$ is $\left[H_{1}^{\mathrm{T}} \mid H_{2}^{\mathrm{T}}\right]^{\mathrm{T}}=\left[\begin{array}{ll|ll}-2 & 0 & 0 & 1\end{array}\right]^{\mathrm{T}}$. We determine $K$ so that the eigenvalues of $\hat{\Xi}+H_{1} K$ are equal to $\{-2,-3\}$. This yields $K=\left[\begin{array}{ll}3.1 & -1.9\end{array}\right]$, so that $\Omega=\hat{\Omega}+H_{2} K=\left[\begin{array}{cc}0 & 0 \\ 7 / 2 & -3 / 2\end{array}\right]$ and $L=-\Omega R_{0, X}^{\dagger}=\left[\begin{array}{ccc}0 & 0 & 0 \\ -7 / 2 & 3 / 2 & 0\end{array}\right]$. Thus, $\sigma\left(A_{X}+B L\right)=\{-1,-2,-3\}$. It is easy to verify that the value of the cost does not change. This solution is optimal, and is also stabilizing. We found a stabilizing optimal control even if $\operatorname{CGCARE}(\Sigma)$ does not admit a stabilizing solution.

\section{REFERENCES}

[1] H. Abou-Kandil, G. Freiling, V. Ionescu and G. Jank. Matrix Riccati Equations in Control and Systems Theory. Birkhäuser, Basel, 2003.

[2] T. Damm. Rational Matrix Equations in Stochastic Control. Lecture Notes in Control and Information Sciences, Vol. 297, Springer Verlag, Berlin, 2004.
[3] V. Dragan, T. Morozan and A.-M. Stoica Mathematical Methods in Robust Control of Discrete-Time Linear Stochastic Systems. Springer, New York, 2010.

[4] A. Ferrante. On the structure of the solution of discrete-time algebraic Riccati equation with singular closed-loop matrix. IEEE Transactions on Automatic Control, AC-49(11):2049-2054, 2004.

[5] L. Ntogramatzidis and A. Ferrante, "The Discrete-Time Generalized Algebraic Riccati Equation: Order Reduction and Solutions' Structure". Systems \& Control Letters, provisionally accepted. Available at http://arxiv.org/pdf/1409.6409v1.pdf.

[6] A. Ferrante, and L. Ntogramatzidis, "The generalized discrete algebraic Riccati equation in linear-quadratic optimal control". Automatica, vol. 49, pp. 471-478, 2013.

[7] A. Ferrante, and L. Ntogramatzidis, "The extended symplectic pencil and the finite-horizon LQ problem with two-sided boundary conditions", IEEE Trans. Aut. Control, vol. 58, pp. 2102-2107, 2013.

[8] A. Ferrante, and L. Ntogramatzidis, "A reduction technique for discrete generalized algebraic and difference Riccati equations", Linear and Multilinear Algebra, vol. 62, pp. 1460-1474, 2014.

[9] A. Ferrante, and L. Ntogramatzidis, "The generalized continuous algebraic Riccati equation and impulse-free continuous-time LQ optimal control". Automatica, vol. 50, pp. 1176-1180, 2014.

[10] A. Ferrante, and L. Ntogramatzidis, "Continuous-time singular Linear-Quadratic control: necessary and sufficient conditions for the existence of regular solutions". Submitted. Available at: http://arxiv.org/abs/1305.5312.

[11] G. Freiling, and A. Hochhaus, "Properties of the solutions of rational matrix difference equations". Comput. Math. Applic., vol. 45, pp. 1137-1154, 2003.

[12] G. Freiling, and A. Hochhaus, "On a class of rational matrix differential equations arising in stochastic control". Linear Algebra Appl., vol. 379, pp. 43-68, 2004.

[13] M.L.J. Hautus and L.M. Silverman. System structure and singular control. Linear Algebra Appl., vol. 50, pp. 369-402, 1983.

[14] R.K. Kalaimani, M.N. Belur and D. Chakraborty "Singular LQ Control, Optimal PD Controller and Inadmissible Initial Conditions". IEEE Trans. Aut. Control, vol. 58, pp. 2603-2608, 2013.

[15] V. Ionescu and C. Oară. Generalized continuous-time Riccati theory. Linear Algebra Appl., vol. 232, pp. 111-130, 1996.

[16] D. Prattichizzo, L. Ntogramatzidis, and G. Marro, "A new approach to the cheap LQ regulator exploiting the geometric properties of the Hamiltonian system". Automatica, vol. 44, pp. 2834-2839, 2008.

[17] A. Saberi and P. Sannuti. Cheap and singular controls for linear quadratic regulators. IEEE Trans. Aut. Control, vol. 32, pp. 208-219, 1987.

[18] A.A. Stoorvogel and A. Saberi. The discrete-time algebraic Riccati equation and linear matrix inequality. Linear Algebra Appl., vol. 274, pp. 317-365, 1998.

[19] H.L. Trentelman, A.A. Stoorvogel, and M. Hautus. Control theory for linear systems. Springer, 2001.

[20] J.C. Willems, A. Kìtapçi, and L.M. Silverman. "Singular optimal control: a geometric approach". SIAM J. Control Optim., vol. 24, pp. 323-337, 1986

\section{APPENDIX A}

In this Appendix, we recall some concepts of classical geometric control theory that are used in this paper. More details can be found e.g. in [19]. Consider an LTI system described by

$$
\begin{aligned}
& \dot{x}(t)=A x(t)+B u(t), \quad x(0)=x_{0} \in \mathbb{R}^{n} \\
& y(t)=C x(t)+D u(t),
\end{aligned}
$$

where $A \in \mathbb{R}^{n \times n}, \quad B \in \mathbb{R}^{n \times m}, \quad C \in \mathbb{R}^{p \times n}$ and $D \in$ $\mathbb{R}^{p \times m}$. We recall that the reachable subspace is $\mathscr{R}_{0}=$ $\operatorname{im}\left[\begin{array}{lllll}B & A B & \ldots & A^{n-1} B\end{array}\right]$, and coincides with the smallest $A$-invariant subspace of $\mathbb{R}^{n}$ containing the image of $B$, i.e. $\mathscr{R}_{0}=\langle A, \operatorname{im} B\rangle$. An output-nulling subspace $\mathscr{V}$ of (14) is a subspace of $\mathbb{R}^{n}$ which satisfies the inclusion

$$
\left[\begin{array}{l}
A \\
C
\end{array}\right] \mathscr{V} \subseteq(\mathscr{V} \oplus\{0\})+\operatorname{im}\left[\begin{array}{c}
B \\
D
\end{array}\right]
$$


which is equivalent to the existence of a matrix $F \in \mathbb{R}^{m \times n}$ such that $(A+B F) \mathscr{V} \subseteq \mathscr{V} \subseteq \operatorname{ker}(C+D F)$. Any real matrix $F$ satisfying these inclusions is referred to as a friend of $\mathscr{V}$. We denote by $\mathfrak{F}(\mathscr{V})$ the set of friends of $\mathscr{V}$. We denote by $\mathscr{V}^{\star}$ the largest output-nulling subspace of (14), which represents the set of all initial states $x_{0}$ of (14) for which a control input exists such that the corresponding output function is identically zero. Such an input function can always be implemented as a static state feedback of the form $u(t)=F x(t)$ where $F \in \mathfrak{F}\left(\mathscr{V}^{\star}\right)$. Eq. (15) is equivalent to the existence of two matrices $\Xi$ and $\Omega$ such that

$$
\left[\begin{array}{l}
A \\
C
\end{array}\right] V=\left[\begin{array}{l}
V \\
0
\end{array}\right] \Xi+\left[\begin{array}{c}
B \\
D
\end{array}\right] \Omega,
$$

where $V$ is a basis matrix for the output-nulling subspace $\mathscr{V}$. The set of solutions of this equation is parameterised in $K_{1}$ as

$$
\left[\begin{array}{l}
\Xi \\
\Omega
\end{array}\right]=\left[\begin{array}{ll}
V & B \\
0 & D
\end{array}\right]^{\dagger}\left[\begin{array}{l}
A \\
C
\end{array}\right] V+\left[\begin{array}{l}
\Phi_{1} \\
\Phi_{2}
\end{array}\right] K_{1},
$$

where $\left[\begin{array}{l}\Phi_{1} \\ \Phi_{2}\end{array}\right]$ is a basis matrix for $\operatorname{ker}\left[\begin{array}{ll}V & B \\ 0 & D\end{array}\right]$. The set of friends $F$ of $\mathscr{V}$ are the solutions of the linear equation $\Omega=-F V$, where $U$ is such that for a certain $\Xi$ the equation (16) holds, and since $V$ is full column-rank, the set of its solutions can be written as

$$
F=-\Omega\left(V^{\top} V\right)^{-1} V^{\top}+K_{2} \Psi,
$$

where $\Psi$ is a full row-rank matrix such that $\operatorname{ker} \Psi=\mathscr{V}$, and $K_{2}$ is arbitrary. Then, the set of friends of $\mathscr{V}$ are parameterised in $K_{1}$ and $K_{2}$, where $K_{1}$ only affects the eigenstructure of the closed-loop restricted to $\mathscr{V}$, i.e., $\sigma(A+$ $B F \mid \mathscr{V})$, whereas $K_{2}$ only affects the eigenstructure $\sigma(A+$ $B F \mid \mathscr{X} / \mathscr{V})$. In other words, given a change of coordinate matrix $T=\left[\begin{array}{ll}T_{1} & T_{2}\end{array}\right]$ where $T_{1}$ is a basis matrix for $\mathscr{V}$, we have $T^{-1}(A+B F) T=\left[\begin{array}{rr}A_{1,1}^{F} & A_{1,2}^{F} \\ 0 & A_{2,2}^{F}\end{array}\right]$, where $A_{1,1}^{F}$ does not depend on $K_{2}$ and $A_{2,2}^{F}$ does not depend on $K_{1}$.

The so-called output-nulling reachability subspace on $\mathscr{V}^{\star}$, herein denoted with $\mathscr{R}^{\star}$, is the smallest $(A+B F)$-invariant subspace of $\mathbb{R}^{n}$ containing the subspace $\mathscr{V}^{\star} \cap B \operatorname{ker} D$, where $F \in \mathfrak{F}\left(\mathscr{V}^{\star}\right)$, i.e., $\mathscr{R}^{\star}=\left\langle A+B F, \mathscr{V}^{\star} \cap B \operatorname{ker} D\right\rangle$ where $F \in$ $\mathfrak{F}\left(\mathscr{V}^{\star}\right)$. Let $F \in \mathfrak{F}\left(\mathscr{V}^{\star}\right)$. The closed-loop spectrum can be partitioned as $\sigma(A+B F)=\sigma\left(A+B F \mid \mathscr{V}^{\star}\right) \cup \sigma(A+$ $\left.B F \mid \mathscr{X} / \mathscr{V}^{\star}\right)$, where $\sigma\left(A+B F \mid \mathscr{V}^{\star}\right)$ is the spectrum of $A+B F$ restricted to $\mathscr{V}^{\star}$ and $\sigma\left(A+B F \mid \mathscr{X} / \mathscr{V}^{\star}\right)$ is the spectrum of the mapping induced by $A+B F$ on the quotient space $\mathscr{X} / \mathscr{V}^{\star}$. The eigenvalues of $A+B F$ restricted to $\mathscr{V}^{\star}$ can be further split into two disjoint sets: the eigenvalues of $\sigma\left(A+B F \mid \mathscr{R}^{\star}\right)$ are all freely assignable with a suitable choice of $F$ in $\mathfrak{F}\left(\mathscr{V}^{\star}\right)$. The eigenvalues in $\sigma\left(A+B F \mid \mathscr{V}^{\star} / \mathscr{R}^{\star}\right)$ are fixed for all the choices of $F$ in $\mathfrak{F}\left(\mathscr{V}^{\star}\right)$. 